\title{
Review: Differential Diagnosis of Drug-Induced Gingival Hyper- plasia and Other Oral Lesions
}

\author{
Einhorn Omer Moshe* \\ Private Dental Office, Israel \\ *Corresponding author: Einhorn Omer Moshe, Private Dental Office, Dr. Einhorn, 89 Medinat Hayehudim \\ street, Herzliya, Israel
}

\begin{abstract}
Chronic medication usage is a major component of the medical diagnosis of patients. Nowadays, some of the most common diseases such as cancer, hypertension, diabetes and etc., are treated with drugs which cause a variety of oral side-effects including gingival over growth and appearance of lesions on the tissues of the oral cavity. As such, drug-induced oral reactions are an ordinary sight in the dental practice. This review will point out the main therapeutic agents causing gingival hyperplasia and other pathologic lesions in the oral cavity. Some frequently used medications, in particular antihypertensives, nonsteroidal anti-inflammatory drugs and even antibiotics, can lead to overgrowth of the gingiva and to the multiple unwanted conditions, namely: Lupus erythematosus, erythema multiforme, mucositis, oral lichen planus, pemphigus vulgaris and more.
\end{abstract}

\section{Keywords}

Drug-induced oral lesions, Gingival hyperplasia, Aphthous stomatitis, Pemphigus vulgaris, Oral lichen planus, Mucositis, Erythema multiforme, Lupus erythematosus, HPV

\section{Introduction}

Drug-induced oral reactions are a common sight in the dental practice. As such, understanding the complete medical history of the patient becomes a crucial diagnostic tool. Proper documentation of systemic and dental conditions of the patient, including past and current administration of medications, is of major significance both clinically and legally. Nowadays, some of the most common diseases such as cancer, hypertension, diabetes, etc. are treated with drugs which cause a variety of oral manifestations. Side-effects resulting from medication usage include salivary dysfunction, gingival overgrowth, oral malodor, mucosal pigmentation, tooth discoloration, alteration of taste sensation and even appearance of lesions on the tissues of the oral cavity. Early recognition and diagnosis of these effects can largely assist in the prevention of further destructive consequences in patients' health status. As life expectancy increases, the number of elderly patients in the dental practice also rises. Individuals of this population are usually subjected to chronic medication intake which requires the clinician to be aware of the various side-effects accompanying these medications. This review will point out the main therapeutic agents causing gingival hyperplasia and other pathologic lesions in the oral cavity. All physicians need a deeper understanding and a true interest in side-effects caused by the intake of various medications, as they can have major implications on both medical status and quality of life of their patients.

\section{Therapeutic Agents Causing Gingival Hyper- plasia}

Gingival hyperplasia is a well-known and common adverse reaction arising from the usage of several medications. Therapeutic agents that cause the enlargement of the gingiva are mostly administered as part of non-dental treatments, in which other tissues, rather than the gingiva, are intended as the target. There are three major groups which are highly associated with this side-effect: Calcium channel blockers, immune suppressants such as Cyclosporine $A$, and anti-epileptics such as Phenytoin. Based on data collected in Australia, out of 114 reported cases, 83 were related to Cyclosporine $A$, calcium channel blockers or Phenytoin [1]. Oral contraceptives are also frequently mentioned when discussing 
this adverse reaction, but to a lesser degree when compared with the previous three. There is a direct correlation between the oral hygiene status of a patient and the severity of drug-induced hyperplasia. Even though maintenance and improvement of oral hygiene is the starting point for any kind of treatment, it does not fully ensure that the condition will be resolved. In severe cases of gingival hyperplasia, when the medical status of the patient enables the clinician, even a complete withdrawal of the medication should be considered. Usually gingival enlargement can be found throughout the oral cavity, yet the overgrowth is mostly prominent at the buccal tissues of both dental arches, and it is initiated at the interdental papilla regions as a painless lesion $[2,3]$. The growth of soft tissues may expand in every direction and as the tissue enlarges it appears to be thickened and lobulated while the epithelial surface is smooth. In advanced stages even the occlusal surfaces of teeth might be covered by the gingiva, which can lead to alterations in several physiological processes such as speech, mastication and occlusion [1,4]. In combination with the presence of a periodontal disease, additional properties would be observable in the gingiva: Prominent red-purple color, high bleeding tendency and a vascularized tissue appearance [4]. According to a clinical review made on the gingival overgrowths, apart from the obvious macroscopic change in the gingival tissue, significant changes in the histology of the tissue are also indicated [1].

\section{Calcium channel blockers}

These agents are extensively used in the treatment of a several cardiovascular diseases. As saliva secretion is closely related to intracellular calcium concentration, why blockers of calcium channels such as Diltiazem, Nifedipine and Verapamil will cause xerostomia and further oral consequences is generally understood [5]. Calcium channel blockers have been known to cause gingival overgrowth since the 1980's [1]. However, since calcium channel blockers are so commonly administered, it is extremely hard to verify the actual incidence of gingival enlargement resulting from the usage of these drugs [4]. An example of the overgrowth induced by calcium channel blockers can be seen in Figure 1 . In a study per-

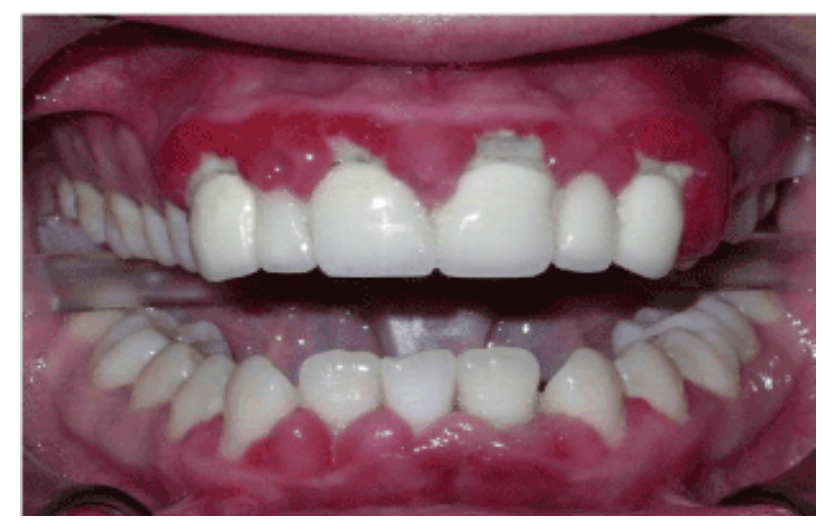

Figure 1: Gingival hyperplasia caused by calcium channel blockers. formed in order to observe the adverse drug reactions of Nifedipine, it has been demonstrated that the drug caused gingival hyperplasia in $20 \%$ of users [2]. Nifedipine acts by inhibiting the process of apoptosis in keratinocytes and prolonging cell life, and as such leading to an epithelial enlargement of the gingiva [6]. Another mechanism of action for Nifedipine has been suggested to be the inhibition of macrophage-induced death of gingival fibroblasts [7]. Another study was performed in order to observe the histological changes generated by Nifedipine in hypertensive animals. Findings have shown alterations in the parotid glands, together with a mild dilation of the vessels and infiltration of inflammatory cells [8].

\section{Phenytoin}

Phenytoin is an anticonvulsant medication. It has been in use for about 80 years and it remains the main agent for the treatment of epilepsy up to this day, as well as being a useful agent in case of cardiac arrhythmias and several neuralgias [1]. It has been reported that $50 \%$ of Phenytoin recipients experience gingival hyperplasia. An even higher percentage is recorded among adolescents. The enlargement of the gingiva usually becomes noticeable within three months after the beginning of the treatment. In most cases, gingival hyperplasia caused by Phenytoin is not resolved with the cessation of treatment [2]. Hyperplastic gingiva as a result of Phenytoin usage can be seen in Figure 2. It has been suggested that the mechanism in which Phenytoin causes gingival overgrowth is by mast-cell mediated androgen stimulation of gingival fibroblasts. As a result of androgen stimulation, further expression of the enzyme $5 \alpha$-reductase takes place within the cells. Findings of a study investigating the mechanisms of phenytoin-induced gingival hyperplasia have suggested that these responses, which take place in gingival fibroblasts, could be a reason to gingival enlargement [9].

\section{Cyclosporine A}

Cyclosporine $A$ (CsA) is an immunosuppressive drug. The main function of this drug is to prevent the rejection of an organ transplant by suppressing the immune system. As high success rates were recorded during renal

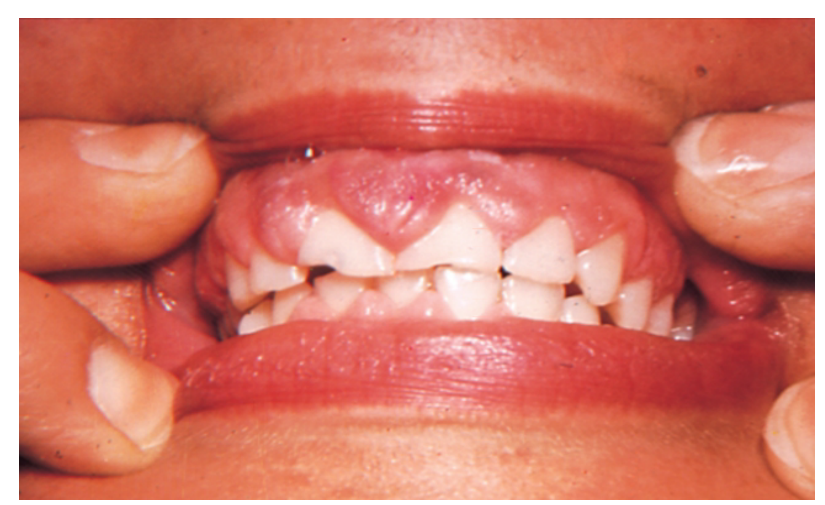

Figure 2: Gingival hyperplasia caused by phenytoin. 


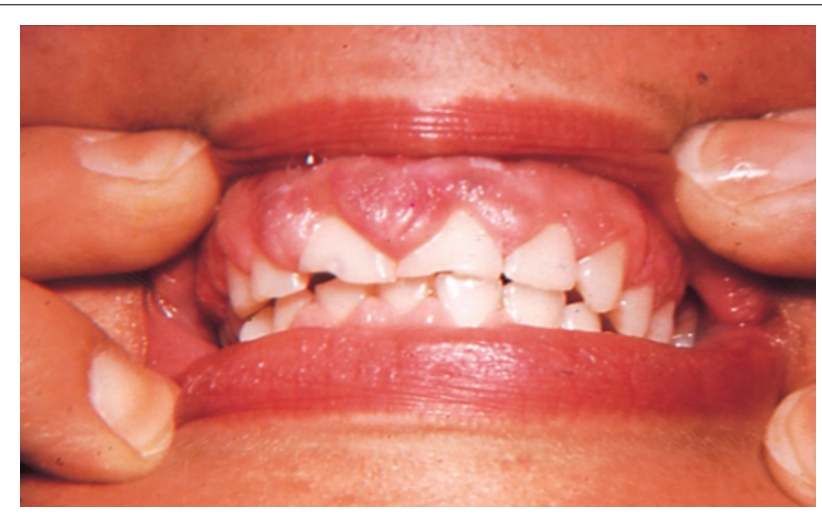

Figure 3: Gingival hyperplasia induced by Cyclosporine A.

transplants, this medication was also provided in transplants of other organs and even in cases of autoimmune diseases [1].

As seen in Figure 3, an overgrowth of gingival tissue by CsA can be attributed both to the enhancement in production of collagen by fibroblasts and to the decrease in collagenase enzyme activity $[2,10]$. As cytokines play an important role in physiological growth and proliferation of tissues, changes in cytokine levels could cause a pathological condition. Under CsA therapy, higher levels of cytokines such as IL- 6 and lower levels of other cytokines including Interferon- $\gamma$ were observed. These alterations in cytokine concentrations promote the synthesis of collagen by fibroblasts, which contributes to gingival enlargement [11]. Another effect of CSA is the upregulation of the keratinocyte growth factor receptor. Keratinocyte growth factor is responsible for the activity and growth of epithelial cells and it has been proven that this growth factor is notably elevated during CsA usage. A study has examined whether an expression of the keratinocyte growth factor receptor is up-regulated in hyperplastic gingival tissue, when compared to normal gingiva. Findings have proved that both receptor-antigen and gene expression levels were elevated in the hyperplastic tissue [12]. Unlike other drug-induced gingival hyperplasia, the one resulting from usage of CsA may exhibit nodular epithelial surface and is more likely to be resolved when treatment has stopped [4]. Even though it is rare, gingival overgrowth caused by CsA can lead to the development of cancerous lesions, such as squamous cell carcinoma and Kaposi's sarcoma [13].

\section{Other Oral Lesions Caused by Therapeutic Agents}

\section{Effects of antibiotic agents on the oral microbiome}

The oral cavity is the starting point of the digestive tract and factors such as temperature, $\mathrm{pH}$ and constant nutrient supply help it serve as a host for the growth of a diverse microbial environment. Thousands of microorganisms including bacteria, fungi and protozoa are physiologically present in the oral cavity. This large group of microorganisms are known as the oral microbiome or oral microflora [14]. The oral microbiome plays a major role in the maintenance of an individual's health status, as it prevents the entrance of exogenous pathogenic microorganisms to the human body. Each site in the oral cavity is characterized by a distinct microflora, matching the biological and physical properties of given site. As such, the microflora present on the tooth surface is widely different than the microflora found at the oral mucous membranes or on the tongue [15]. The stability of the oral microbiome can be highly affected by changes in oral $\mathrm{pH}$ and salivary flow rate, smoking and the intake of antibiotic agents. As bacteria are the most numerous members of the oral microflora, any alteration in the physiological concentrations of these microorganisms can result in the formation of large bacterial colonies, known as dental plaque. Dental plaque is essentially a biofilm covering the surfaces of the tooth and it is responsible for the production of metabolites leading to oral diseases, such as dental caries and periodontal disease. The goal of antibiotic therapy is to eradicate, or at least slow down the growth of harming bacteria. Unfortunately, when using these agents in the treatment of bacterial infections, members of the oral microflora are also affected, causing a change in the oral environment. As antibiotic agents damage the integrity of the oral microflora, the defense of the oral cavity becomes compromised. This state allows exogenous pathogenic microorganisms to reside and colonize in the oral cavity, which usually leads to oral diseases. Another undesirable consequence of antibiotic treatment is opportunistic infections caused by members of the oral microflora. Under normal circumstances, members of the oral microflora are harmless, and they contribute to the physiological functions of the oral cavity. As the oral microbiome is being altered by antibiotic agents, these microorganisms can cause severe infections with harmful oral and systemic manifestations [16]. Streptococci is the most common species of bacteria that normally colonizes the oral cavity, as seen in Figure 4. Other main bacterial species usually found in the oral cavity include Lactobacillus, Actinomyces, Selenomonas, Veillonella, Fusobacterium, Treponema and Gemella. Although they are members of the oral microflora, most of these species can become major constituents of dental plaque and contribute to the manifestation of dental caries and periodontal diseases [17].

\section{Aphthous lesions}

Recurrent aphthous stomatitis, seen in Figure 5, is a chronic condition appearing mostly during adolescence, that may reappear during adulthood with reduced severity. A study examining the relationship between the appearance of aphthous lesions and medication intake has indicated that NSAIDs (Nonsteroidal Anti-Inflammatory Drugs) and $\beta$-blockers were both agents that may cause this type of oral lesions $[18,19]$. Alendronate, an agent used in the treatment of osteoporosis and bone diseases, has also been reported to cause aphthous lesions in the oral cavity [20]. The exact mechanism in which these agents act is still unclear. 


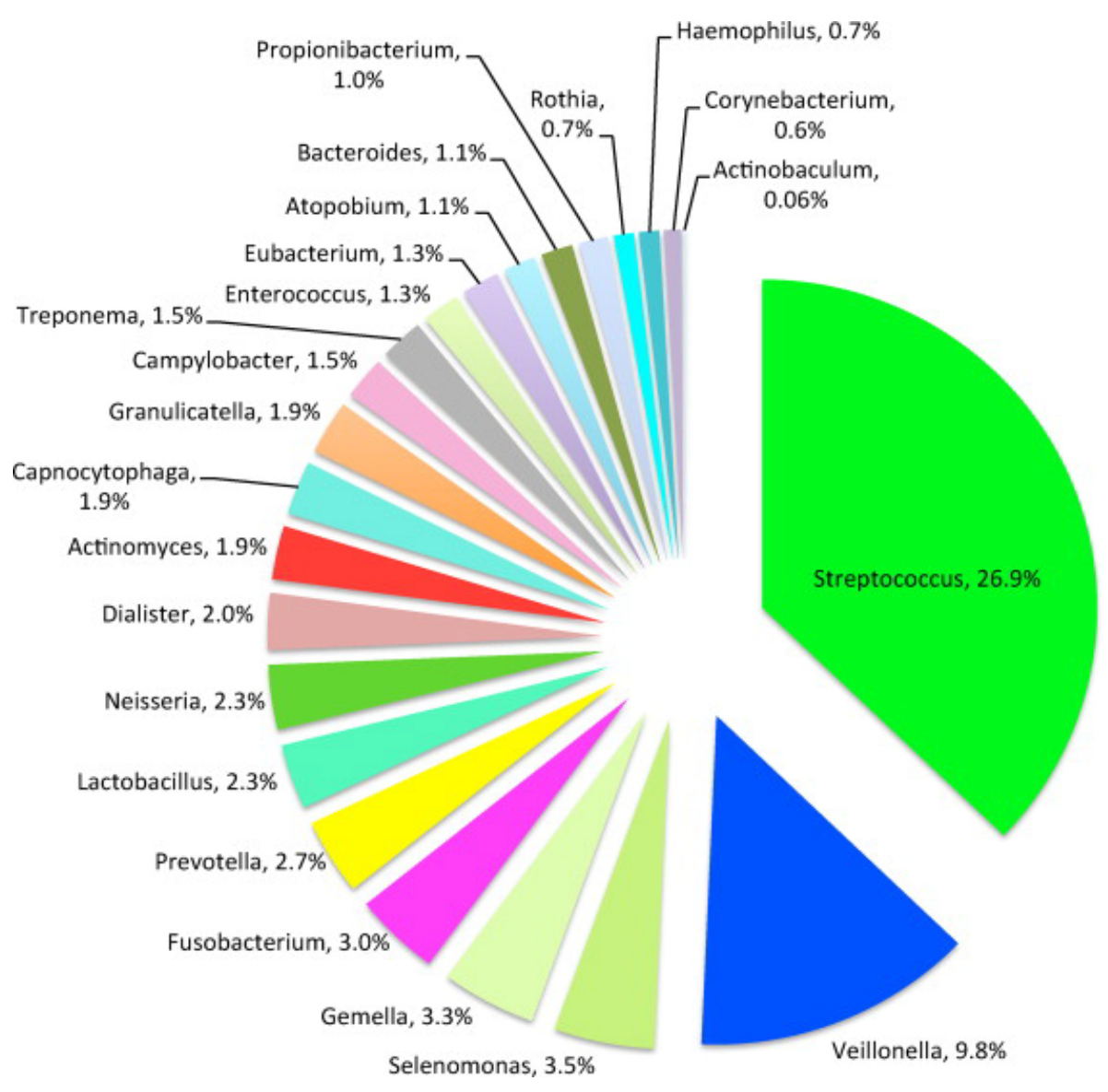

Figure 4: Composition of oral microbiome.

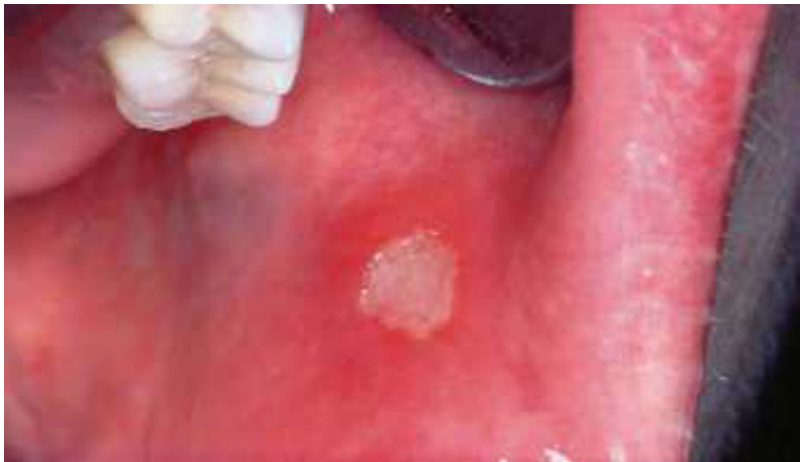

Figure 5: Aphthous lesion on the buccal mucosa, caused by NSAIDs.

\section{Pemphigoid and bullous lesions}

Pemphigoid lesions in the oral cavity are autoimmune in origin and can be described as blisters arising from the oral mucosa, as seen in Figure 6. Autoantibodies directed against bullous pemphigoid antigens on the hemidesmosomes are factors causing this side-effect. This interaction results in tissue separation between the epithelium and the connective tissue. Several therapeutic agents have been reported to trigger the reaction between the autoantibodies and the hemidesmosomes in the oral cavity. Some of the main agents related with this side-effect are NSAIDs, penicillin-derivative antibiotics and cardiovascular agents such as ACE (Angio-

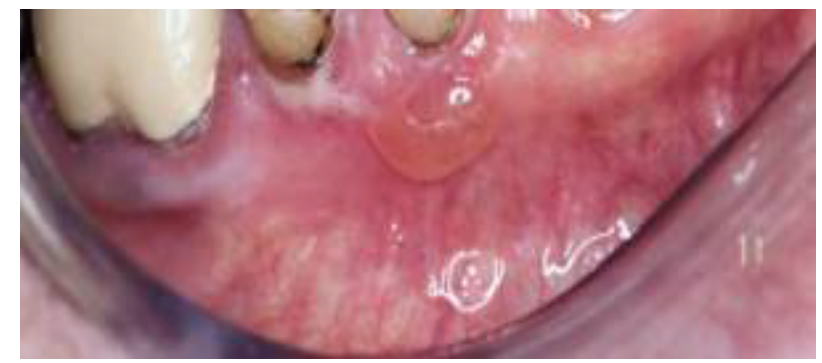

Figure 6: Pemphigoid lesion in the oral cavity, caused by NSAIDs.

tensin-Converting-Enzyme) inhibitors and Furosemide $[13,21]$. Vancomycin, an antibiotic agent, has been reported to cause manifestations of the sub-epidermal bullous disease, the linear Immunoglobulin A (IgA) disease. The main effect of this disease is the linear deposition of IgA at the basement membrane, resulting in pemphigoid and bullous lesions on the oral mucosa [22].

\section{Pemphigus}

Pemphigus is a severe chronic autoimmune mucocutaneous disease. $90 \%$ of pemphigus cases are classified as pemphigus vulgaris. Pemphigus vulgaris is characterized by the appearance of blisters, ulcers and erosions on the oral mucosa, as seen in Figure 7. The mechanism of action for this condition involves Immunoglobulin $G$ autoantibodies produced against Cadherin, an adhesion 


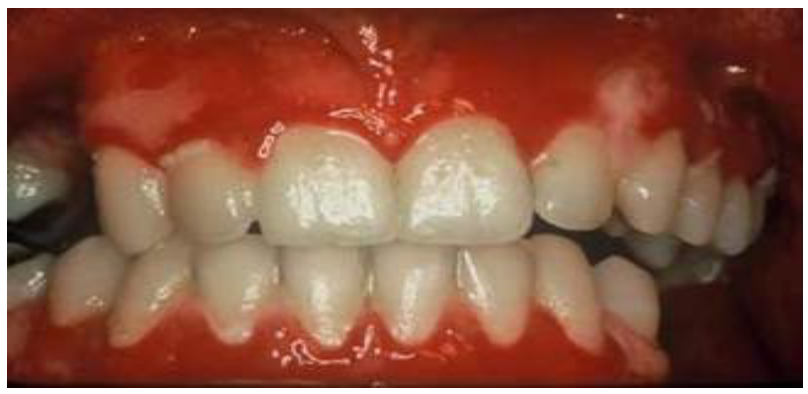

Figure 7: Pemphigus vulgaris-ruptured blisters and ulcers on oral mucosa.

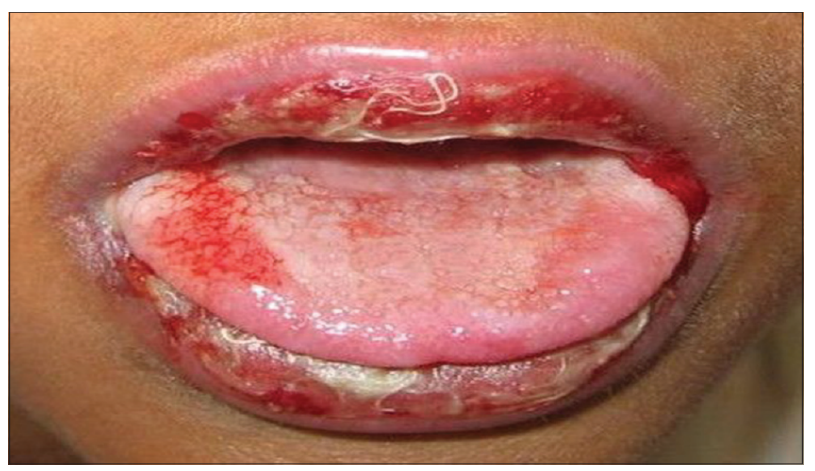

Figure 8: Oral manifestation of erythema multiforme.

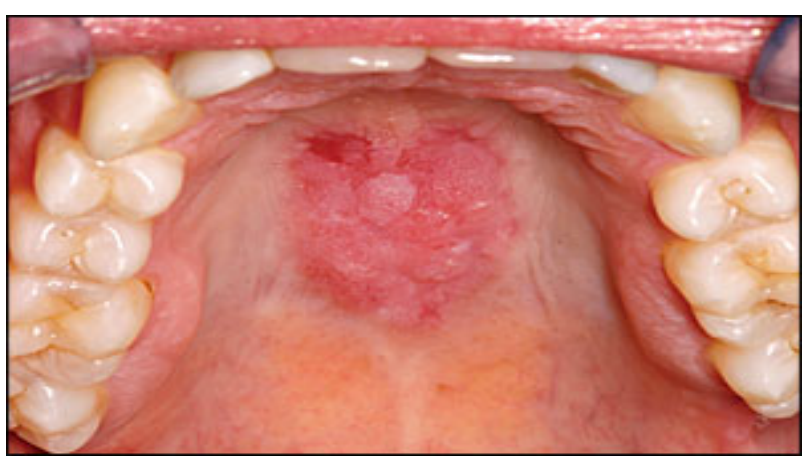

Figure 9: Oral manifestation of systemic lupus erythematosus.

molecule. The interaction between the autoantibodies and Cadherin results in acantholysis, a loss of cell-to-cell adhesion, leading to separation in the mucosa. Blisters arising from this condition rupture rapidly, usually before the patient's arrival to the dental practice, leaving painful ulcers on the mucosa. Drugs mostly reported to cause these effects were Rifampicin, Diclofenac and ACE inhibitors [23-25].

\section{Erythema multiforme}

Erythema multiforme is an acute, self-limited, recurring condition that is considered as a type III-IV hypersensitivity reaction. Erythema multiforme, seen in Figure 8 , is characterized by typical target-like skin lesions and oral ulcers. Oral lesions are present as small vesicles that rupture in 2-3 days and leave painful erosions covered by necrotic pseudo-membrane. Some of the therapeutic agents related with the appearance of

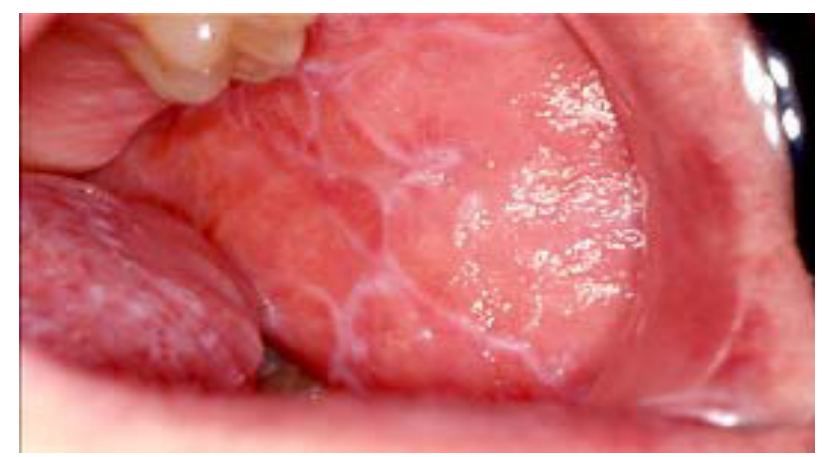

Figure 10: Reticular form of lichenoid lesion, located on the buccal mucosa.

this lesions include cephalosporins, NSAIDs and tetracyclines [13].

\section{Lupus erythematosus}

Lupus erythematosus is an autoimmune disease that can result from prolonged drug exposure and manifest as oral lesions, seen in Figure 9. It has been suggested that individuals suffering from this condition are genetically predisposed, although it remains unclear what triggers this specific autoimmune reaction. One study suggests that certain drugs lead to over-expression of lymphocyte function-associated antigen 1 which results in lupus erythematosus [26]. Drugs related with this disease are antihypertensive and anti-arrhythmic agents, such as Hydralazine and Procainamide. Isoniazid, an antibiotic agent for the treatment of tuberculosis, is also known to cause this side-effect. Lupus can be further classified as an acute, subacute or a chronic condition. Subacute cutaneous lupus is related with drugs such as calcium channel blockers, ACE inhibitors, and thiazides. Drug-induced chronic cutaneous lupus is associated with 5-flurouracil and NSAIDs [27]. Lupus lesions are most commonly seen on the palate and the buccal mucosa.

\section{Lichenoid lesions}

Oral lichen planus is a chronic mucocutaneous disease of an unknown cause, characterized by the appearance of lesions in the oral mucosa. Lichen planus lesions are typically present as bilateral white lesions, sometimes with ulcers, mostly in the area of the buccal mucosa and sometimes on the tongue. Even though the etiology is not completely known, it has been suggested that an immunologically mediated process is taking place, mimicking a hypersensitivity reaction. This condition also exhibits CD4 and CD8 T-cells infiltrates at the epithelial-connective tissue junction resulting in keratinocyte destruction by the T-cells. It has been indicated that the severity of symptoms is often related to the patient's level of stress. Oral lichen planus lesions are subclassified according to their form: Reticular, plaque, erythematous, erosive or bullous. The most common type of lichenoid lesions is the reticular form, known as 'Wickham's straie'. It can be observed in Figure 10 


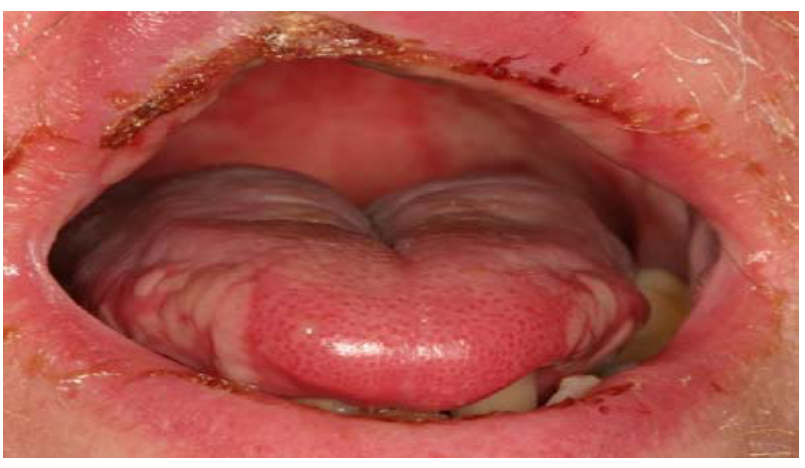

Figure 11: Mucositis as a result of radiation and chemotherapy.

$[28,29]$. According to a study gathering reports of cases exhibiting the manifestation of oral lichen planus, the most common medications causing this drug-induced oral reaction are antihypertensives and anti-arrhythmic agents such as $\beta$-blockers, ACE inhibitors, methyldopa and quinidine, as well as several antimalarial agents. NSAIDs have also been mentioned as agents causing this side-effect. The same study has also indicated that it is extremely hard to differentiate between drug-induced lichen planus and the idiopathic appearance of this disease $[30,31]$.

\section{Mucositis}

Mucositis is defined as a state of severe inflammation accompanied with the appearance of lesions and ulcers in the mucosa of the oral cavity, as seen in Figure 11. Intense bleeding and pain are very common in this condition [32]. Mucositis is a major side-effect of radiation and chemotherapy, and the agents leading to this effect are cytotoxic drugs such as 5-flurouracil, Methotrexate and Cytarabine. The incidence of this condition is around $40 \%$ among patients undergoing standard chemotherapy. Mucositis is an end-result of several combined effects, leading to epithelial dysfunction. Events like free radical injury, DNA damage, cell cycle arrest and disturbance of cell interactions are all results of chemo- and radiotherapy, eventually resulting in inflammation of the oral mucosa [32]. A study investigating the pathophysiology in which the cytotoxic drugs act has found that both Methotrexate and 5-flurouracil administration have led to histological change of tissues in the oral cavity [33]. A further consequence of the immunosuppressive effect of these drugs is the invasion of pathological microorganisms, leading to severe infections. Some of these organisms can be gram-negative bacteria, Mycobacterium Tuberculosis and fungi species. Criteria such as age and diet of the patient, oral hygiene maintenance during treatment and the type of cancer, are all considered to be risk factors and have a principal effect on the development of mucositis [34].

\section{Lesions arising as a consequence of immunosup- pression}

Oral candidiasis arises as a result of a broad-spec-

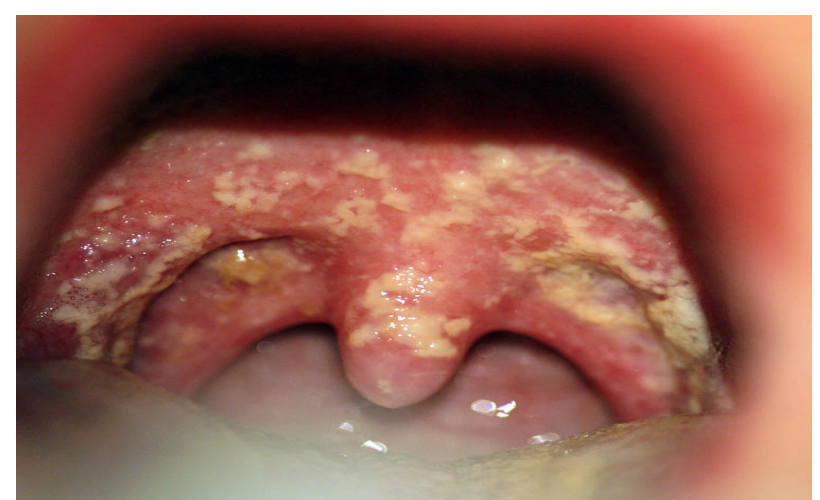

Figure 12: Oral candidiasis, white pseudo-membrane on the palate.

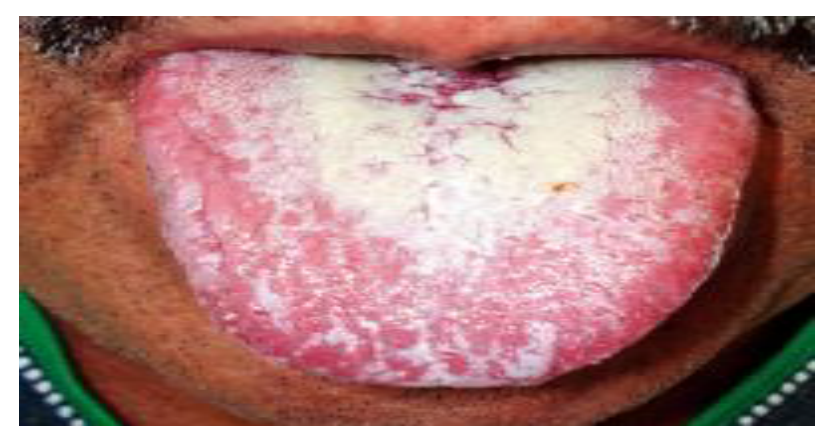

Figure 13: Oral candidiasis, white pseudo-membrane on the tongue.

trum antibiotic usage or by treatment with immunosuppressive agents and cytotoxic drugs. Candida Albicans is an opportunistic pathogen, responsible for $95 \%$ of oral fungal infections. This pathogen is normally found in oral, gastro-intestinal and genital regions, taking advantage of immunologic dysfunction in order to further multiply. This condition is characterized by the appearance of a white pseudo-membrane on the tongue, buccal mucosa or palate, seen in Figure 12 and Figure 13. As the white membrane can be scraped off, it is a way to differentiate this lesion from other white lesions of the oral cavity [35].

Other pathogens that can cause an infection in the oral cavity as a consequence of immunosuppressive therapy are the HPV (Human Papilloma Virus) and viruses of the Herpes family such as HSV (Herpes Simplex Virus), EBV (Epstein-Barr Virus) and HHV-8 (Human Herpes Virus 8). HPV is a major etiological factor for the manifestation of pre-cancerous lesions and conditions in the oral cavity such as verruca vulgaris. Examples for the oral manifestation of HPV infections can be seen in Figure 14 and Figure 15.

EBV infection can lead to conditions such as Burkitt's lymphoma and hairy leukoplakia (Figure 16), whereas HHV-8 gives rise to Kaposi's sarcoma (Figure 17), a tumor that can grow anywhere in the orofacial region.

\section{Discussion and Conclusions}

An immense number of therapeutic agents can 


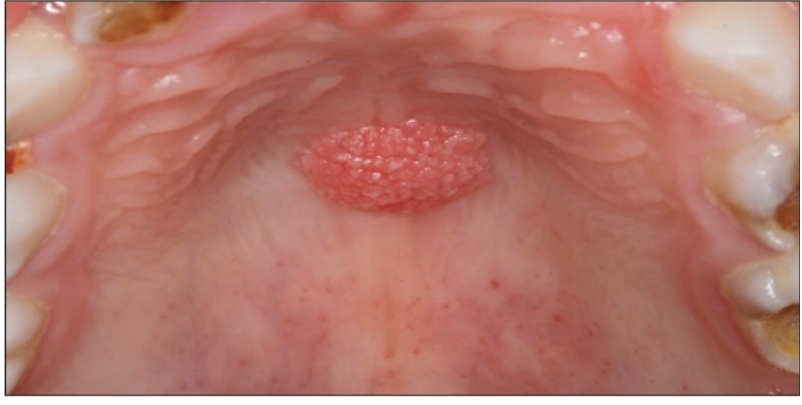

Figure 14: Oral manifestation of HPV infection, papilloma on the palate.

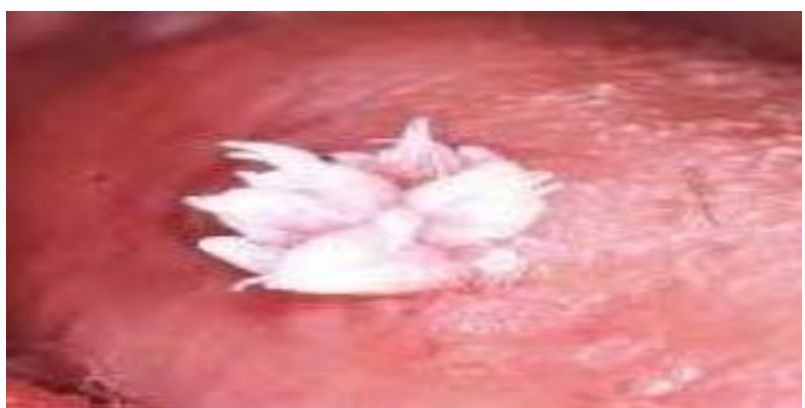

Figure 15: Verrucous vulgaris-white, verrucous-shaped papilloma on the tongue.

cause undesirable oral side-effects and many of these agents are integrated into the treatment of very common diseases and pathological conditions. As such, drug-induced oral reactions are a common complaint in the dental practice [3]. Most drug-induced oral side-effects are mild to moderate when examining their severity. These adverse reactions can frequently interfere with several physiological functions such as mastication, digestion, taste perception and occlusion, as well with aesthetic appearance. However, the usage of certain medications may become a major risk factor regarding the patient's general health, and result in more dangerous consequences such as the appearance of pre-cancerous lesions [36]. In most cases, the treatment of drug-induced oral reactions involves the withdrawal and replacement of the agent in question, along with a symptomatic therapy. This can usually resolve the oral manifestation, but unfortunately cessation or replacement of the medication is not always an option. As such, the dental physician should always follow the general guidelines that include an extensive knowledge of possible complications, careful patient monitoring and proper case management. The symptomatic treatment of drug-induced oral reactions and the possible substitution or withdrawal of medication causing these reactions are important, but they are not the only steps of the treatment. Maintenance of good oral hygiene, cessation of bad habits such as alcohol consumption and smoking, adequate nutrition-especially of vitamins, and proper hydration can all influence the extent of the undesirable effects resulting from medication usage [37]. Most drug-induced oral side-effects are directly cor-

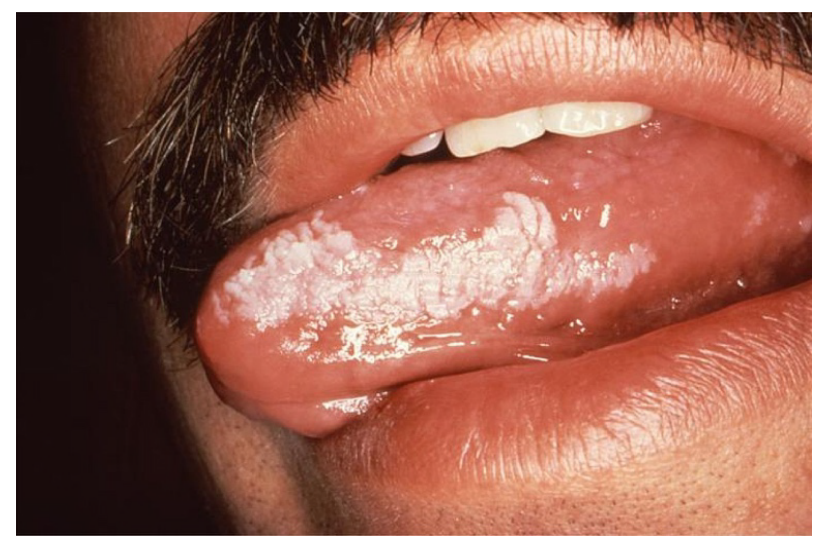

Figure 16: Hairy leukoplakia, as a result of EBV infection.

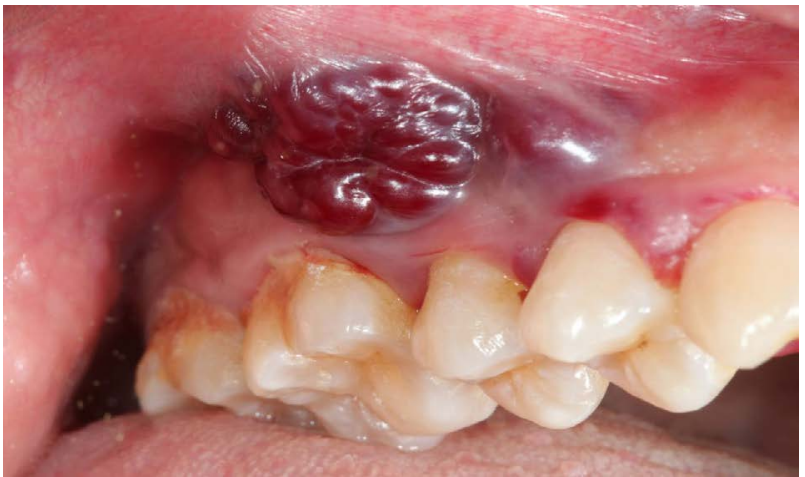

Figure 17: Kaposi's sarcoma in the oral cavity.

related to oral hygiene and oral health status. The presence of either periodontal diseases or plaque retentive factors can contribute to the extent of these reactions and therefore they need to be treated. An important role of the dentist is to instruct the patient on the correct maintenance of oral hygiene. Proper brushing technique of both teeth and tongue, along with instructions for interdental cleaning and mouthwash usage should be a part of the explanation. Both satisfactory treatment and oral hygiene instructions can significantly improve patient's motivation and social life [38]. In the case of gingival overgrowth, it has been noted that effective plaque control has a vital role in the success of the treatment, along with medication substitution. Upon failure of these measures, surgical intervention might be needed even though it does not assure prevention of recurrence [1]. Certain oral lesions require not only a clinical inspection, but also histologic confirmation. A study investigating the accuracy of diagnoses made by dentists has examined 976 cases in which diagnoses determined by clinical inspections was compared with histologic specimens. Results have shown that $43 \%$ of the cases were misdiagnosed, including the misdiagnosis of cancerous lesions in $5.6 \%$ of the time. The study has concluded that all excised lesions should undergo histologic confirmation [39]. Lastly, it is important to mention the rapid progress in the field of pharmacology. Such progress makes all clinicians pay special attention to the therapeutic agents used by their patients. 
It is necessary to understand both the mechanisms in which the drugs act, as well as the possible adverse effects caused by its usage [36].

\section{References}

1. Marshall RI, Bartold PM (1999) A clinical review of drug induced gingival overgrowths. Aust Dent J 44: 219-232.

2. Abdollahi M, Radfar M (2003) A review of drug-induced oral reactions. J Contemp Dent Pract 4: 10-31.

3. Trophimus GJ (2014) The effect of drugs in the oral cavity-A review. J Pharm Sci \& Res 6: 89-96.

4. Barbara Anne Taylor (2003) Management of drug-induced gingival enlargement. Aust Prescr 26: 11-13.

5. Hattori T, Wang PL (2007) Calcium antagonists cause dry mouth by inhibiting resting saliva secretion. Life Sci 81 : 683-690.

6. Shimizu Y, Kataoka M, Seto H, Kido J, Nagata T (2002) Nifedipine induces gingival epithelial hyperplasia in rats through inhibition of apoptosis. J Periodontol 73: 861-867.

7. Fujimori $Y$, Maeda $S$, Saeki M, Morisaki I, Kamisaki $Y$ (2001) Inhibition by nifedipine of adherence-and activated macrophage-induced death of human gingival fibroblasts. Eur J Pharmacol 415: 95-103.

8. Nikos Seferosa, Ioanna Daskalaa, Antonia Kotsioub, Madeleine Tsamouric, Christine Tesseromatis, et al. (2014) Nifedipine-induced histological changes in the parotid glands of hypertensive rats. Braz Oral Res 28: 1-5.

9. Soory M, Suchak A (2001) The effects of human mast-cell products and of phenytoin on androgen $5 a$-reductase expression in human gingival fibroblasts. Arch Oral Biol 46: 847-855.

10. Schincaglia GP, Forniti F, Cavallini R, Piva R, Calura G, et al. (1992) Cyclosporin A increases type I procollagen production and mRNA level in human gingival fibroblasts in vitro. J Oral Pathol Med 21: 181-185.

11. Theofilos TM, Gerard JL, John JM, Chris RI (1999) Cyclosporin a regulates Interleukin $1 B$ and Interleukin 6 expression in gingiva: Implications for gingival overgrowth. J Periodontol 70: 294-300.

12. Das SJ, Newman HN, Olsen I (2002) Keratinocyte growth factor receptor is up-regulated in cyclosporin A-induced gingival hyperplasia. J Dent Res 81: 683-687.

13. Scully C, Bagan JV (2004) Adverse drug reactions in the orofacial region. Crit Rev Oral Biol Med 15: 221-239.

14. Wade WG (2013) The oral microbiome in health and disease. Pharmacol Res 69: 137-143.

15. Philip D Marsh (2000) Role of the oral microflora in health. MEHD 12: 130-137.

16. Marsh PD, Percival RS (2006) The oral microflora-friend or foe? Can we decide? IDJ 56: 233-239.

17. Palmer RJ (2014) Composition and development of oral bacterial communities. Periodontol 2000 64: 20-39.

18. Boulinguez S, Reix S, Bedane C, Debrock C, Bouyssou-Gauthier ML, et al. (2000) Role of drug exposure in aphthous ulcers: A case-control study. Br J Dermatol 143: 1261-1265.

19. Crispian Scully, Meir Gorsky, Francina Lozada Nur (2002) Aphthous ulcerations. Dermatologic Therapy 15: 185-205.

20. Gonzalez-Moles MA, Bagan-Sebastian JV (2000) Alendro- nate related oral mucosa ulcerations. J Oral Pathol Med 29: 514-518.

21. Vassileva S (1998) Drug-induced pemphigoid: Bullous and cicatricial. Clin Dermatol 16: 379-387.

22. Palmer RA, Ogg G, Allen J, Banerjee A, Ryatt KS, et al. (2001) Vancomycin induced linear IgA disease with autoantibodies to BP180 and LAD285. Br J Dermatol 145: 816820.

23. Gange RW, Rhodes EL, Edwards CO, Powell MEA (1976) Pemphigus induced by rifampicin. $\mathrm{Br} \mathrm{J}$ Dermatol 95: 445448.

24. Matz H, Bialy-Golan A, Brenner S (1997) Diclofenac: A new trigger of pemphigus vulgaris? Dermatology 195: 48-49.

25. Ruocco V, Satriano RA, Guerrera V (1992) "Two step" pemphigus induction by ACE inhibitors. Int J Dermatol 31: 33-36.

26. Yung R, Powers D, Johnson K, Amento E, Carr D, et al. (1996) Mechanisms of drug-induced lupus. II. T cells overexpressing lymphocyte function-associated antigen 1 become autoreactive and cause a lupuslike disease in syngeneic mice. J Clin Invest 97: 2866-2871.

27. Vedove CD, Del Giglio M, Schena D, Girolomoni G (2009) Drug-induced lupus erythematosus. Arch Dermatol Res 301: 99-105.

28. Roopashree MR, Gondhalekar RV, Shashikanth MC, George J, Thippeswamy SH, et al. (2010) Pathogenesis of oral lichen planus-A review. J Oral Pathol Med 39: 729-734.

29. Scully C, el-Kom M (1985) Lichen planus: Review and update on pathogenesis. J Oral Pathol 14: 431-458.

30. Thompson DF, Skaehill PA (1994) Drug induced lichen planus. Pharmacotherapy 14: 561-571.

31. Van Dis ML, Parks ET (1995) Prevalence of oral lichen planus in patients with diabetes mellitus. Oral Surg Oral Med Oral Pathol Oral Radiol Endod 79: 696-700.

32. Duncan M, Grant G (2001) Oral and intestinal mucositis-causes and possible treatments. Aliment Pharmacol Ther 18: 853-874.

33. Logan RM, Stringer AM, Bowen JM, Gibson RJ, Sonis ST, et al. (2009) Is the pathobiology of chemotherapy-induced alimentary tract mucositis influenced by the type of mucotoxic drug administered? Cancer Chemother Pharmacol 63: 239-251.

34. Maddireddy URN, Gogula VR, Pingali Usha Rani, lyyapu KM, Avula Suman, et al. (2004) Chemotherapy-induced and/or radiation therapy-induced oral mucositis-complicating the treatment of cancer. Neoplasia 6: 423-431.

35. Soysa NS, Samaranayake LP, Ellepola AN (2004) Cytotoxic drugs, radiotherapy and oral candidiasis. Oral Oncol 40: 971-978.

36. Abdollahi M, Rahimi R, Radfar M (2008) Current opinion on drug-induced oral reactions: A comprehensive review. J Contemp Dent Pract 9: 1-15.

37. Ciçek Y, Ertaş U (2003) The normal and pathological pigmentation of oral mucous membrane: A review. J Contemp Dent Pract 4: 76-86.

38. Armstrong BL, Sensat ML, Stoltenberg JL (2010) Halitosis: A review of current literature. J Dent Hyg 84: 65-74.

39. Kondori I, Mottin RW, Laskin DM (2011) Accuracy of dentists in the clinical diagnosis of oral lesions. Quintessence Int 42: 575-577. 